\title{
Association between Active School Travel and Depressive Symptoms among 51,702 Adolescents in 26 Low- and Middle-Income Countries
}

\author{
Shuan Liu ${ }^{1}$, Sitong Chen ${ }^{2}$, Xiaohong Zhu ${ }^{3}$, Brendon Stubbs, Qian Yu ${ }^{5}$, Mark D. Griffiths ${ }^{6}$, Can Jiao \\ Aiguo Chen ${ }^{8}$, Md Mahbub Hossain ${ }^{9}$, Zsolt Demetrovics ${ }^{10,11}$, Albert S. Yeung ${ }^{12}$, Jinming Li $^{5}$, \\ Xingyu Zhang ${ }^{5}$ and Liye $\mathrm{Zou}^{5, *}$ \\ ${ }^{1}$ Faculty of Educational Science, South China Normal University, Guangzhou, 510631, China \\ ${ }^{2}$ Institute for Health and Sport, Victoria University, Melbourne, 8001, Australia \\ ${ }^{3}$ College of Sports Science, Jishou University, Jishou, 212013, China \\ ${ }^{4}$ Department of Psychological Medicine, Institute of Psychiatry, Psychology and Neuroscience, King's College London, London, \\ WC2R 2LS, UK \\ ${ }^{5}$ Exercise and Mental Health Laboratory, Institute of KEEP Collaborative Innovation, Shenzhen University, Shenzhen, \\ 518060, China \\ ${ }^{6}$ Psychology Department, Nottingham Trent University, Nottingham, NG1 4FQ, UK \\ ${ }^{7}$ School of Psychology, Shenzhen University, Shenzhen, 518060, China \\ ${ }^{8}$ College of Physical Education, Yangzhou University, Yangzhou, 225127, China \\ ${ }^{9}$ Department of Health Promotion and Community Health Sciences, School of Public Health, Texas A\&M University, College \\ Station, TX77843, USA \\ ${ }^{10}$ Institute of Psychology, ELTE Eötvös Loránd University, Budapest, 1064, Hungary \\ ${ }^{11}$ Centre of Excellence in Responsible Gaming, University of Gibraltar, Europa Point, GX11 1AA, Gibraltar \\ ${ }^{12}$ Depression Clinical and Research Program, Massachusetts General Hospital, Boston, MA 02114, USA \\ *Corresponding Author: Liye Zou. Email: liyezou123@gmail.com
}

Received: 23 February 2021 Accepted: 04 April 2021

\begin{abstract}
Little is known about the role of active school travel (AST) on mental health among adolescents. Thus, this study aimed to explore the AST-depression association among adolescents aged 12-15 years from 26 low- and middleincome countries (LMICs). Data from the Global School-based Student Health Survey were analyzed in 51,702 adolescents [mean (SD) age 13.8 (1.0) years; $49.3 \%$ boys). Both depressive symptoms and AST were assessed by a single question self-reported measure, respectively. Participants who reported having 5 days or above were considered as AST. Multivariable logistic regression analysis (accounting for sampling weights) was performed while controlling for gender, age, physical activity, sedentary behavior, and food insecurity, and a countrywide meta-analysis was undertaken. The prevalence of depressive symptoms and AST were 30.1\% and $37.0 \%$, respectively. Compared with those not having AST, adolescents with AST were less likely to have self-reported depressive symptoms $(\mathrm{OR}=0.88,95 \% \mathrm{CI}$ : 0.85-0.93) regardless of gender. Countrywide meta-analysis demonstrated that having AST versus not having AST was associated with $12 \%$ lower odds for depressive symptoms $(\mathrm{OR}=0.88$; 95\% CI: 0.82-0.94) but with a moderate between-country heterogeneity $\left(I^{2}=59.0 \%\right)$. Based on large samples of adolescents from LMICs, it would be expected that AST may play a critical role in preventing adolescent depression worldwide. However, it is necessary to consider more country-specific
\end{abstract}


factors when implementing AST-related mental health interventions. Future studies should adopt the solid study design to confirm or negate our research findings.

\section{KEYWORDS}

Active travel; depression; Global School-based Health Survey; low- and middle-income countries

\section{Introduction}

Adolescent depression is a common mood disorder that causes loss of interest, sleep deprivation, substance use disorder, feelings of sadness, and suicidal ideation, which can interfere with daily activities and academic performance among children and adolescents [1,2]. Data from the Global Disease Burden has indicated that the global prevalence of depressive symptoms is relatively high worldwide [1,2], for example, $7.1 \%-19.4 \%$ in 11 European countries (adolescent sample) [3]. Adolescent depression is associated with multiple adverse outcomes and preventing/managing this during adolescence is of great importance to adults' mental health [4]. A meta-analysis of 11 pooled cohorts found that adolescents with depression had 2.78 times increased odds of depression in their adulthood [4]. There is an increasing recognition that non-genetic risk factors play an integral role in the development of depression and may offer a potential opportunity to prevent depression [5].

Physical activity (PA) has been increasingly recognized as a part of healthy lifestyle behaviors that can protect against depressive symptoms among adolescents [6-10]. Furthermore, converging evidence consistently shows that an increase in PA level is associated with a reduced level of depressive symptoms. For example, a Norwegian study indicated that physically active adolescents were more likely to have a lower probability of being depressed, after controlling for some sociodemographic and other lifestyle behavior factors [11]. Similar results were also found in a study using population-based sample of Canadian adolescents (controlling for age, sex, ethnicity, and parental education) [12]. These crosssectional studies with self-report data are further corroborated by a seminal longitudinal study using accelerometers to measure PA level (total PA was recorded as count per minute [CPM]) at around 12 years, 14 years, and 16 years of age [13]. More specifically, researchers found lower odds for depression scores were associated with a $15 \mathrm{~min} /$ day increase in moderate-to-vigorous PA in adolescents (adjusted odd ratio $<1$ ) [11].

Active school travel (AST), which refers to, generally, human-powered transport modes, such as walking, cycling, wheeling, scootering, or skateboarding, to travel between school and home [14], as one essential source of PA, has received increasing attention in recent years and has been investigated in association with psychological well-being among adolescents. In adults, a systematic review indicated the positive roles of active travel in reducing depression, but such topic among adolescents requires further investigation $[15,16]$. Against this background, researchers have theoretically proposed that a negative association between depression level and AST may be attributed to two things [17]. First, AST provides youths with the opportunity to engage in activities that can directly increase PA level [18], which could be, in turn, linked with decreased depressive symptoms [19]. Second, as a daily behavior, AST occurs commonly and easily, which has been associated with positive affection and reduced negative emotions due to social interaction and environmental relationships. In particular, adolescents with lower socioeconomic status are more likely to have AST relative to their counterparts [20-23]. Presumably, adolescents from LMICs are more likely to engage in AST. Within this theoretical foundation, a study using Chinese nationally representative samples found that participants with AST had lower odds of being depressed [24]. Recently, a cross-sectional study in Shanghai City (China) also supported the 
assumption that AST may reduce depressive symptoms among adolescents in grades 7-9 [19], whereas adolescents without AST were more likely to be depressed [19]. Such findings have been replicated in Canadian adolescents [25].

Admittedly, the most noticeable limitation is that previous studies' findings were based on single country or city [19,24], which limits the generalization because of being conducted in a specific setting [26]. Consequently, multinational studies would allow researchers gain insights into the relationships between AST and depressive symptoms, offering insights concerning public health messages for mental well-being promotion at a more global level. As suggested by a review [17], the AST role in promoting mental health may vary by social and geographical factors. It is, therefore, expected that pattern on the relationship between AST and depressive symptoms may be different across countries or regions. Indeed, a multinational study would allow comparison between various nations so that more specific policies can be adopted for mental health promotion among the adolescents. Last but not least, there is limited evidence on associations between AST and mental health (depression) from low- and middle-income countries (LMICs). Owing to income disparity across these countries, motorized lifestyles including being driven to school may not be an option. Instead, adolescents in LMICs are more likely to be active commuters between home and school (i.e., walking or cycling to school). In this regard, if the association between AST and depressive symptom among those who are in LMICs can be evaluated, more effective and economical ways to prevent depressive symptoms can be identified accordingly.

To address these aforementioned gaps in the literature, the present study explored the association between AST and depressive symptoms among the adolescents of 26 LMICs using the Global Schoolbased Health Survey (GSHS) data. It was hypothesized that adolescents with AST would be less likely to have self-reported depressive symptoms.

\section{Methods}

\subsection{Study Survey}

Publicly available data from the Global School-based Student Health Survey (GSHS) were analyzed. Details on this survey can be found at http://www.who.int/chp/gshs and http://www.cdc.gov/gshs. Briefly, the GSHS was jointly developed by the World Health Organization and the United States Centers for Disease Control and Prevention and other United Nations allies. The core aim of the survey was to assess and quantify risk and protective factors of major non-communicable diseases. The survey used a standardized two-stage probability sampling design for the selection process within each participating country. For the first stage, schools were selected with probability proportional to size sampling. The second stage involved the random selection of classrooms which included students aged 12-15 years within each selected school. All students in the selected classrooms were eligible to participate in the survey regardless of age. To provide high quality data and reduce the bias during data collection, surveys of each country were standardized and comparable across countries, and well-trained research staff conducted the survey, in cooperation with local education department and medical administration.

Data collection was performed during one regular class period. The questionnaire was translated into the local language in each country and consisted of multiple-choice response options. Students recorded their response on computer scanned sheets. All GSHS surveys were approved, in each country, by both a national government administration (most often the Ministry of Health or Education) and an institutional review board or ethics committee. Student privacy was protected through anonymous and voluntary participation, and informed consent was obtained as appropriate from the students, parents, and/or school officials. Data were weighted for non-response and probability selection. Country survey having variables of active school travel and depressive symptoms were included into this study $(n=26$; after excluding 4 countries that either AST or depressive symptoms not assessed). Because the GSHS is an iterative survey study with updates every 4 or 5 years, the latest dataset of each survey was included in the present analysis (Year: 2003-2008). 


\subsection{Active School Travel (Exposure)}

Active school travel was assessed by one single question: "How many days did you walk or ride a bicycle to and from school during the past 7 days?" Participants self-reported their responses according to their actual situations (responses: 0-7 days as integer). Participants having 5 days or more for walking or cycling were regarded as being active, whereas those not reporting 5 days or more for walking or cycling were regarded as being passive. The rational for selecting 5 days or more is that adolescents normally have 5 days for schooling per week, which is in line with a previous study [27].

\subsection{Depressive Symptom (Outcome)}

Depressive symptoms were assessed by one single question "During the past 12 months, did you ever feel so sad or hopeless almost every day for two weeks or more in a row that you stopped doing your usual activities?" Responses were yes and no. Participants answering yes were considered as having depressive symptoms, in line with previous studies [26].

\subsection{Control Variables}

Gender [19,24,26], age [19,24,26], physical activity [19,24,26], sedentary behavior [19,24,26], and food insecurity [26] were regarded as covariates based on previously published research. To assess levels of physical activity, a reliable and valid measure was used [28]. The questions asked about the number of days with physical activity of at least $60 \mathrm{~min}$ during the past 7 days and during a typical week [28]. This did not include physical activity during physical education or gym classes. The number of active days during the past week and a typical week were averaged, in line with previous studies [26]. Sedentary behavior was assessed with the question "How much time do you spend during a typical or usual day sitting and watching television, playing computer games, talking with friends, or doing other sitting activities?" with answer options: $<1,1-2,3-4,5-6,7-8$, and $\geq 8$ h/day. Food insecurity was assessed by the question "During the past 30 days, how often did you go hungry because there was not enough food in your home?" The answers consisted of always, most of the time, sometimes, rarely, and never.

\subsection{Statistical Analysis}

All the statistical analyses were performed with SPSS 25.0 and R. For descriptive analyses, SPSS was used to calculate the prevalence of AST and depressive symptoms, respectively. The prevalence of AST and depressive symptoms by gender and country was also calculated. Multivariable logistic regression analysis was implemented by SPSS to assess the association between AST and depressive symptoms (overall, by gender and country). The variables of AST and depressive symptoms were binary. The regression was analysis was adjusted for age, gender, physical activity, sedentary behavior, and food insecurity, as well as country, with the exception of the gender-stratified and country-wise analyses which were not adjusted for gender and country, respectively.

This meta-analytic method was evaluated via RStudio (1.4.1103). To assess the level of between-country heterogeneity, the Higgin's $I^{2}$ statistic was calculated based on country-wise estimates using R. This represents the degree of heterogeneity that is not explained by sampling error with a value of $<40 \%$ often considered as negligible and $40 \%-60 \%$ as moderate heterogeneity [29]. A pooled estimate was obtained by combining the estimates for each country into a random effect meta-analysis. All variables were included in the regression analyses as categorical variables with the exception of age and PA (continuous variables). Under $3.8 \%$ of the data were missing for all the variables used in the analysis. Complete case analysis was done. Taylor linearization methods were used in all analyses to account for the sample weighting and complex study design. Within the GSHS protocol, weighting accounted for the probability of selection of schools and classrooms, non-responding schools and students. Results from the logistic regression analyses are presented as odds ratios with $95 \%$ confidence intervals (CIs). The statistical significance level was $p<0.05$. 


\section{Results}

The final sample comprised 51,702 adolescents aged 12-15 years with a mean age of 13.8 years $(\mathrm{SD}=1.0)$ and $49.3 \%$ were males $(\mathrm{Tab} .1)$. More information concerning covariates can be found in Tab. 1 as well. The individual samples from different study countries were surveyed from 2003 to 2008 with response rates ranging 69\% (Uganda) to 100\% (Jordan). The overall prevalence of depressive symptoms was $30.1 \%$, while the prevalence of active school travel (active 5 or more days) was 37.0\% (see Tab. 1). Tab. 2 shows the prevalence of active school travel (active) and depressive symptoms (yes) of each country samples. More specifically, the prevalence of depressive symptoms by country ranged from $16.6 \%$ (Thailand) to $51.4 \%$ (Zambia), and the prevalence of active school travel for each country ranged from $14.4 \%$ (St Lucia) to $75.8 \%$ (China).

Table 1: Sample characteristics in this study

\begin{tabular}{|c|c|c|c|c|}
\hline & & \multicolumn{2}{|c|}{ Overall sample } & \multirow[t]{2}{*}{ Weighted (\%) } \\
\hline & & $\mathrm{n}$ & $\%$ & \\
\hline Total & & 51702 & 100 & l \\
\hline \multicolumn{5}{|l|}{ Gender } \\
\hline & Boy & 24765 & 47.9 & 49.3 \\
\hline & Girl & 26937 & 52.1 & 50.7 \\
\hline Age $($ mean $\pm \mathrm{SD})$ & & 13.8 & 1.0 & $13.8 \pm 0.9$ \\
\hline \multicolumn{5}{|l|}{ Food insecurity } \\
\hline & Never & 28643 & 55.4 & 50.1 \\
\hline & Rarely & 9358 & 18.1 & 21.2 \\
\hline & Sometimes & 10237 & 19.8 & 22.5 \\
\hline & Most of the time & 2171 & 4.2 & 4.1 \\
\hline & Always & 1293 & 2.5 & 2.1 \\
\hline \multicolumn{5}{|l|}{ Sedentary behavior } \\
\hline & Less than $1 \mathrm{~h}$ per day & 17527 & 33.9 & 31.9 \\
\hline & 1 to $2 \mathrm{~h}$ per day & 18147 & 35.1 & 36.4 \\
\hline & 3 to $4 \mathrm{~h}$ per day & 9306 & 18.0 & 19.3 \\
\hline & 5 to $6 \mathrm{~h}$ per day & 3154 & 6.1 & 6.5 \\
\hline & 7 to $8 \mathrm{~h}$ per day & 1241 & 2.4 & 2.3 \\
\hline & More than $8 \mathrm{~h}$ per day & 2327 & 4.5 & 3.6 \\
\hline Physical activity (mean \pm SD) & & 3.4 & 2.3 & $3.4 \pm 2.2$ \\
\hline \multicolumn{5}{|l|}{ Active school travel } \\
\hline & Passive & 33865 & 65.5 & 63.0 \\
\hline & Active & 17837 & 34.5 & 37.0 \\
\hline \multicolumn{5}{|l|}{ Depressive symptoms } \\
\hline & Negative & 35726 & 69.1 & 69.9 \\
\hline & Positive & 15976 & 30.9 & 30.1 \\
\hline
\end{tabular}


Table 2: The prevalence of active school travel and depressive symptoms by country

\begin{tabular}{llllll}
\hline Country & $\mathrm{N}$ & Year & Response rate (\%) & Active school travel (\%) & Depressive symptoms (\%) \\
\hline Argentina & 1403 & 2007 & 77.1 & 55.4 & 29.6 \\
Botswana & 1252 & 2005 & 95 & 29.0 & 39.4 \\
Chile & 1900 & 2004 & 85 & 49.8 & 30.8 \\
China & 2126 & 2003 & 99 & 75.8 & 18.3 \\
Djibouti & 913 & 2007 & 83 & 35.4 & 37.7 \\
Ecuador & 1716 & 2007 & 85.6 & 17.8 & 25.4 \\
Egypt & 4475 & 2006 & 87 & 37.0 & 35.3 \\
Grenada & 1127 & 2007 & 78 & 16.8 & 23.2 \\
Guyana & 980 & 2004 & 80 & 18.7 & 32.8 \\
India & 6553 & 2007 & 83 & 42.1 & 24.4 \\
Indonesia & 2939 & 2007 & 93.1 & 46.8 & 21.0 \\
Jordan & 1490 & 2007 & 100 & 30.4 & 37.1 \\
Kenya & 2403 & 2005 & 84 & 25.6 & 47.9 \\
Morocco & 1838 & 2006 & 84 & 24.4 & 37.2 \\
Philippines & 3792 & 2003 & 79 & 22.2 & 40.2 \\
Seychelles & 969 & 2007 & 82 & 29.4 & 32.1 \\
Sri Lanka & 2388 & 2008 & 89 & 41.9 & 31.8 \\
St Lucia & 1000 & 2007 & 82 & 14.1 & 23.3 \\
St Vincent & 1022 & 2007 & 84 & 19.9 & 27.5 \\
Tanzania & 1635 & 2006 & 87 & 16.8 & 24.0 \\
Thailand & 2490 & 2008 & 93 & 28.0 & 16.6 \\
Tunisia & 2391 & 2008 & 83 & 50.5 & 36.1 \\
Uganda & 1646 & 2003 & 69 & 29.1 & 39.7 \\
Uruguay & 421 & 2006 & 71.2 & 69.4 & 51.4 \\
Venezuela & 1867 & 2004 & 86 & 14.5 & \\
Zambia & 966 & 2004 & 70.0 & & 22.0 \\
\hline & & & & 19.2 & \\
\hline
\end{tabular}

Tab. 3 presents the results from multivariable logistic regression. Results for crude model can be found in Tab. 3. Results showed that adolescents with AST were less likely to have depressive symptoms $(\mathrm{OR}=0.88,95 \% \mathrm{CI}: 0.85-0.93, p<0.001)$ after adjusting for age, gender, PA, SB, and food insecurity as well as country. Similarly, for male adolescents, those with AST had lower odds ratio for depressive symptoms $(\mathrm{OR}=0.84,95 \% \mathrm{CI}: 0.79-0.90, p<0.005)$ after adjustment for age, PA, SB, and food insecurity as well as country. There was also a significant association among female adolescents $(\mathrm{OR}=$ 0.92, 95\%CI: $0.87-0.98, p<0.01)$.

The country-wise association between active school travel and depressive symptoms is shown in Fig. 1. The pooled estimate from a meta-analysis for all the samples was 0.88 (95\% CI: 0.82-0.94) with a moderate level of between-country heterogeneity $\left(I^{2}=59.8 \%\right)$. There was significant heterogeneity between country groups $(p=0.000)$. 
IJMHP, 2021, vol.23, no.2

Table 3: Association between active school travel and depressive symptoms estimated by multivariable logistic regression (overall and by gender)

\begin{tabular}{|c|c|c|c|c|c|c|c|c|c|c|c|c|c|c|c|c|c|c|}
\hline & \multicolumn{6}{|c|}{ Overall } & \multicolumn{6}{|c|}{ Boys } & \multicolumn{6}{|c|}{ Girls } \\
\hline & \multicolumn{3}{|c|}{ Crude model } & \multicolumn{3}{|c|}{ Adjusted model } & \multicolumn{3}{|c|}{ Crude model } & \multicolumn{3}{|c|}{ Adjusted model } & \multicolumn{3}{|c|}{ Crude model } & \multicolumn{3}{|c|}{ Adjusted model } \\
\hline & OR & \multicolumn{2}{|c|}{$95 \% \mathrm{CI}$} & OR & \multicolumn{2}{|c|}{$95 \% \mathrm{CI}$} & OR & \multicolumn{2}{|c|}{$95 \% \mathrm{CI}$} & OR & \multicolumn{2}{|c|}{$95 \% \mathrm{CI}$} & OR & \multicolumn{2}{|c|}{$95 \% \mathrm{CI}$} & OR & \multicolumn{2}{|c|}{$95 \% \mathrm{CI}$} \\
\hline \multicolumn{19}{|l|}{$\begin{array}{l}\text { Active school } \\
\text { travel }\end{array}$} \\
\hline Passive & \multicolumn{3}{|c|}{ Reference group } & \multicolumn{3}{|c|}{ Reference group } & \multicolumn{3}{|c|}{ Reference group } & \multicolumn{3}{|c|}{ Reference group } & \multicolumn{3}{|c|}{ Reference group } & \multicolumn{3}{|c|}{ Reference group } \\
\hline Active & 0.89 & 0.84 & 0.96 & 0.88 & 0.85 & 0.93 & 0.78 & 0.70 & 0.87 & 0.84 & 0.79 & 0.90 & 0.88 & 0.77 & 0.96 & 0.92 & 0.87 & 0.98 \\
\hline \multicolumn{19}{|l|}{ Gender } \\
\hline Boy & 0.86 & 0.80 & 0.93 & 0.83 & 0.77 & 0.90 & 1 & & & & & & I & & & & & \\
\hline Girl & \multicolumn{3}{|c|}{ Reference group } & \multicolumn{3}{|c|}{ Reference group } & & & & & & & & & & & & \\
\hline \multicolumn{19}{|l|}{$\begin{array}{l}\text { Food } \\
\text { insecurity }\end{array}$} \\
\hline Never & 0.48 & 0.40 & 0.58 & 0.49 & 0.41 & 0.60 & 0.46 & 0.36 & 0.59 & 0.47 & 0.36 & 0.62 & 0.46 & 0.36 & 0.59 & 0.51 & 0.40 & 0.65 \\
\hline Rarely & 0.59 & 0.49 & 0.72 & 0.64 & 0.52 & 0.78 & 0.62 & 0.48 & 0.81 & 0.69 & 0.52 & 0.91 & 0.52 & 0.40 & 0.67 & 0.59 & 0.45 & 0.76 \\
\hline Sometimes & 0.78 & 0.64 & 0.94 & 0.80 & 0.66 & 0.98 & 0.76 & 0.58 & 0.99 & 0.75 & 0.55 & 1.02 & 0.73 & 0.57 & 0.95 & 0.84 & 0.66 & 1.08 \\
\hline $\begin{array}{l}\text { Most of the } \\
\text { time }\end{array}$ & 1.15 & 0.93 & 1.41 & 1.03 & 0.84 & 1.27 & 1.09 & 0.80 & 1.50 & 0.99 & 0.72 & 1.36 & 1.13 & 0.86 & 1.48 & 1.06 & 0.81 & 1.38 \\
\hline Always & \multicolumn{3}{|c|}{ Reference group } & Refer & rence $g$ & group & Refer & rence & roup & Refer & ence $g$ & group & Refer & rence $\mathrm{g}$ & group & Refer & rence & roup \\
\hline $\begin{array}{l}\text { Sedentary } \\
\text { behavior }\end{array}$ & & & & & & & & & & & & & & & & & & \\
\hline $\begin{array}{l}\text { Less than } 1 \mathrm{~h} \\
\text { per day }\end{array}$ & 0.67 & 0.57 & 0.79 & 0.62 & 0.53 & 0.72 & 0.67 & 0.55 & 0.81 & 0.62 & 0.49 & 0.79 & 0.68 & 0.54 & 0.86 & 0.61 & 0.49 & 0.76 \\
\hline $\begin{array}{l}1 \text { to } 2 \mathrm{~h} \text { per } \\
\text { day }\end{array}$ & 0.56 & 0.48 & 0.65 & 0.57 & 0.49 & 0.66 & 0.53 & 0.43 & 0.65 & 0.55 & 0.44 & 0.69 & 0.59 & 0.48 & 0.73 & 0.59 & 0.48 & 0.72 \\
\hline $\begin{array}{l}3 \text { to } 4 \mathrm{~h} \text { per } \\
\text { day }\end{array}$ & 0.68 & 0.58 & 0.80 & 0.70 & 0.60 & 0.83 & 0.63 & 0.52 & 0.78 & 0.67 & 0.53 & 0.85 & 0.72 & 0.58 & 0.89 & 0.72 & 0.58 & 0.90 \\
\hline $\begin{array}{l}5 \text { to } 6 \mathrm{~h} \text { per } \\
\text { day }\end{array}$ & 0.70 & 0.60 & 0.82 & 0.74 & 0.62 & 0.88 & 0.69 & 0.54 & 0.86 & 0.72 & 0.55 & 0.93 & 0.71 & 0.56 & 0.90 & 0.76 & 0.59 & 0.97 \\
\hline $\begin{array}{l}7 \text { to } 8 \mathrm{~h} \text { per } \\
\text { day }\end{array}$ & 0.84 & 0.63 & 1.11 & 0.80 & 0.57 & 1.12 & 0.77 & 0.54 & 1.11 & 0.74 & 0.48 & 1.16 & 0.92 & 0.65 & 1.30 & 0.85 & 0.58 & 1.23 \\
\hline $\begin{array}{l}\text { More than } 8 \mathrm{~h} \\
\text { per day }\end{array}$ & Refer & ence $g$ & group & Refer & ence $g$ & group & Refer & rence & roup & Refe & ence $g$ & group & Refer & rence $g$ & group & Refer & rence & roup \\
\hline $\begin{array}{l}\text { Physical } \\
\text { activity } \\
(\text { mean } \pm \text { SD) }\end{array}$ & 0.96 & 0.94 & 0.98 & 0.97 & 0.96 & 0.99 & 0.93 & 0.89 & 0.95 & 0.96 & 0.94 & 0.98 & 0.96 & 0.93 & 0.98 & 0.99 & 0.96 & 0.99 \\
\hline $\begin{array}{l}\text { Age (mean } \pm \\
\text { SD) }\end{array}$ & 1.15 & 1.10 & 1.21 & 1.14 & 1.09 & 1.19 & 1.16 & 1.10 & 1.21 & 1.14 & 1.08 & 1.20 & 1.16 & 1.08 & 1.25 & 1.13 & 1.06 & 1.21 \\
\hline Country & & & & & & & & & & & & & & & & & & \\
\hline
\end{tabular}

(Continued) 
Table 3 (continued).

\begin{tabular}{|c|c|c|c|c|c|c|c|c|c|c|c|c|c|c|c|c|c|c|}
\hline \multirow[b]{4}{*}{ Argentina } & \multicolumn{6}{|c|}{ Overall } & \multicolumn{6}{|c|}{ Boys } & \multicolumn{6}{|c|}{ Girls } \\
\hline & \multicolumn{3}{|c|}{ Crude model } & \multicolumn{3}{|c|}{ Adjusted model } & \multicolumn{3}{|c|}{ Crude model } & \multicolumn{3}{|c|}{ Adjusted model } & \multicolumn{3}{|c|}{ Crude model } & \multicolumn{3}{|c|}{ Adjusted model } \\
\hline & \multirow{2}{*}{$\frac{\mathrm{OR}}{0.40}$} & \multicolumn{2}{|c|}{$95 \% \mathrm{CI}$} & \multirow{2}{*}{$\frac{\text { OR }}{0.60}$} & \multicolumn{2}{|c|}{$95 \% \mathrm{CI}$} & \multirow{2}{*}{$\frac{\text { OR }}{0.32}$} & \multicolumn{2}{|c|}{$95 \% \mathrm{CI}$} & \multirow{2}{*}{$\frac{\mathrm{OR}}{0.5}$} & \multicolumn{2}{|c|}{$95 \% \mathrm{CI}$} & \multirow{2}{*}{$\begin{array}{l}\text { OR } \\
0.48\end{array}$} & \multicolumn{2}{|c|}{$95 \% \mathrm{CI}$} & \multirow{2}{*}{$\frac{\text { OR }}{0.69}$} & \multicolumn{2}{|c|}{$95 \% \mathrm{CI}$} \\
\hline & & 0.31 & 0.52 & & 0.45 & 0.81 & & 0.22 & 0.46 & & 0.32 & 0.78 & & 0.35 & 0.66 & & 0.49 & 0.96 \\
\hline Botswana & 0.61 & 0.50 & 0.75 & 0.72 & 0.56 & 0.91 & 0.63 & 0.45 & 0.88 & 0.74 & 0.5 & 1.12 & 0.63 & 0.49 & 0.80 & 0.69 & 0.52 & 0.91 \\
\hline Chile & 0.42 & 0.33 & 0.53 & 0.71 & 0.54 & 0.95 & 0.28 & 0.20 & 0.38 & 0.48 & 0.32 & 0.71 & 0.62 & 0.45 & 0.86 & 0.98 & 0.70 & 1.38 \\
\hline China & 0.21 & 0.17 & 0.26 & 0.36 & 0.28 & 0.46 & 0.25 & 0.18 & 0.34 & 0.42 & 0.29 & 0.61 & 0.20 & 0.15 & 0.25 & 0.32 & 0.24 & 0.42 \\
\hline Djibouti & 0.57 & 0.46 & 0.71 & 0.76 & 0.60 & 0.98 & 0.59 & 0.43 & 0.81 & 0.77 & 0.53 & 1.11 & 0.59 & 0.43 & 0.81 & 0.75 & 0.53 & 1.05 \\
\hline Ecuador & 0.32 & 0.25 & 0.42 & 0.54 & 0.41 & 0.71 & 0.29 & 0.20 & 0.41 & 0.47 & 0.31 & 0.7 & 0.38 & 0.27 & 0.53 & 0.61 & 0.43 & 0.86 \\
\hline Egypt & 0.52 & 0.40 & 0.67 & 0.87 & 0.66 & 1.15 & 0.49 & 0.35 & 0.69 & 0.78 & 0.52 & 1.16 & 0.59 & 0.41 & 0.84 & 0.98 & 0.68 & 1.39 \\
\hline Grenada & 0.29 & 0.23 & 0.36 & 0.38 & 0.29 & 0.49 & 0.28 & 0.20 & 0.40 & 0.36 & 0.24 & & 0.30 & 0.22 & 0.40 & 0.39 & 0.28 & 0.54 \\
\hline Guyana & 0.46 & 0.36 & 0.59 & 0.61 & 0.48 & 0.78 & 0.42 & 0.28 & 0.62 & 0.54 & 0.36 & 0.81 & 0.50 & 0.39 & 0.64 & 0.67 & 0.51 & 0.87 \\
\hline India & 0.31 & 0.25 & 0.38 & 0.52 & 0.41 & 0.66 & 0.34 & 0.25 & 0.47 & 0.57 & 0.39 & 0.83 & 0.28 & 0.22 & 0.37 & 0.46 & 0.34 & 0.61 \\
\hline Indonesia & 0.25 & 0.20 & 0.32 & 0.36 & 0.28 & 0.46 & 0.26 & 0.19 & 0.37 & 0.37 & 0.25 & 0.53 & 0.26 & 0.19 & 0.34 & 0.36 & 0.27 & 0.48 \\
\hline Jordan & 0.56 & 0.43 & 0.72 & 0.74 & 0.56 & 0.97 & 0.41 & 0.29 & 0.59 & 0.53 & 0.35 & 0.79 & 0.73 & 0.53 & 1.01 & 0.94 & 0.67 & 1.32 \\
\hline Kenya & 0.87 & 0.72 & 1.05 & 1.12 & 0.89 & 1.40 & 0.97 & 0.72 & 1.33 & 1.29 & 0.89 & 1.88 & 0.82 & 0.64 & 1.04 & 0.99 & 0.75 & 1.30 \\
\hline Morocco & 0.56 & 0.45 & 0.70 & 0.80 & 0.62 & 1.03 & 0.54 & 0.38 & 0.76 & 0.72 & 0.48 & 1.08 & 0.62 & 0.48 & 0.80 & 0.88 & 0.66 & 1.15 \\
\hline Philippines & 0.64 & 0.52 & 0.78 & 0.81 & 0.64 & 1.02 & 0.64 & 0.47 & 0.86 & 0.76 & 0.52 & 1.1 & 0.66 & 0.51 & 0.84 & 0.84 & 0.65 & 1.10 \\
\hline Seychelles & 0.45 & 0.38 & 0.53 & 0.56 & 0.46 & 0.69 & 0.43 & 0.32 & 0.56 & 0.5 & 0.36 & 0.71 & 0.48 & 0.39 & 0.60 & 0.61 & 0.49 & 0.77 \\
\hline Sri Lanka & 0.44 & 0.35 & 0.55 & 0.74 & 0.58 & 0.94 & 0.52 & 0.36 & 0.75 & 0.86 & 0.57 & 1.29 & 0.40 & 0.31 & 0.51 & 0.64 & 0.48 & 0.85 \\
\hline St Lucia & 0.29 & 0.23 & 0.36 & 0.38 & 0.29 & 0.50 & 0.24 & 0.17 & 0.34 & 0.31 & 0.2 & 0.48 & 0.34 & 0.25 & 0.46 & 0.44 & 0.32 & 0.60 \\
\hline St Vincent & 0.36 & 0.28 & 0.45 & 0.49 & 0.38 & 0.64 & 0.31 & 0.21 & 0.45 & 0.44 & 0.29 & 0.68 & 0.42 & 0.31 & 0.57 & 0.53 & 0.39 & 0.73 \\
\hline Tanzania & 0.30 & 0.24 & 0.36 & 0.51 & 0.40 & 0.65 & 0.38 & 0.27 & 0.52 & 0.6 & 0.41 & 0.87 & 0.25 & 0.19 & 0.34 & 0.44 & 0.33 & 0.60 \\
\hline Thailand & 0.19 & 0.15 & 0.24 & 0.27 & 0.21 & 0.35 & 0.23 & 0.16 & 0.32 & 0.33 & 0.22 & 0.48 & 0.17 & 0.13 & 0.22 & 0.23 & 0.17 & 0.31 \\
\hline Tunisia & 0.54 & 0.43 & 0.66 & 0.86 & 0.67 & 1.09 & 0.48 & 0.35 & 0.66 & 0.77 & 0.52 & 1.12 & 0.62 & 0.47 & 0.80 & 0.94 & 0.71 & 1.24 \\
\hline Uganda & 0.62 & 0.50 & 0.77 & 0.84 & 0.66 & 1.05 & 0.65 & 0.46 & 0.91 & 0.86 & 0.58 & 1.28 & 0.63 & 0.49 & 0.82 & 0.81 & 0.62 & 1.06 \\
\hline Uruguay & 0.20 & 0.15 & 0.28 & 0.33 & 0.23 & 0.47 & 0.13 & 0.06 & 0.28 & 0.25 & 0.11 & 0.55 & 0.26 & 0.17 & 0.39 & 0.39 & 0.26 & 0.58 \\
\hline Venezuela & 0.27 & 0.22 & 0.32 & 0.46 & 0.37 & 0.58 & 0.26 & 0.19 & 0.37 & 0.44 & 0.3 & 0.65 & 0.28 & 0.22 & 0.36 & 0.48 & 0.37 & 0.62 \\
\hline Zambia & \multicolumn{3}{|c|}{ Reference group } & \multicolumn{3}{|c|}{ Reference group } & \multicolumn{3}{|c|}{ Reference group } & Refe & rence & group & Refe & rence $g$ & group & Refer & rence $g$ & group \\
\hline
\end{tabular}

Note: $\mathrm{OR}=$ Odds ratio; $\mathrm{CI}=$ confidence interval.

\section{Discussions}

To our knowledge, the present study is one of the few to explore the association between AST and depressive symptoms among adolescents and is the first to examine the association more globally among LMICs. The study found that adolescents engaging in AST had lower odds of depressive symptoms across the countries examined. Concerning the national difference of association between AST and depressive symptoms, the present study indicates a moderate inconsistency.

As the first multinational investigation confirming a negative association between AST and depressive symptoms among adolescents, the findings are supported by other single-country or regional study samples $[19,24]$. From a global perspective, AST may play an important role in reducing depressive symptoms among adolescents, which offers implications for policymakers and researchers. Although the mechanism associating AST and depressive symptoms remains unknown, some plausible interpretations can be proposed. One major behavioral explanation is that AST improves overall PA level [18]. Higher levels of PA have been recognized as a driver in lower depressive symptoms [6,7,30]. Collectively, adolescents with AST appear to have sufficient PA, which may reduce their depressive symptoms. Additionally, AST 
is a type of behavior which interacts with social and environmental surroundings (e.g., children walking to school may be doing so with their friends), which may also lead to reduced negative emotions [31] and positive mood [25]. On this point, reduced negative emotion and increased positive mood may give rise to decreased depressive symptoms among adolescents. The third explanation may be relevant to stress that originates from biopsychosocial changes during the adolescence. AST may be a good way to release stress [32] and can also improve fitness [18] that can regulate stress responses [32]. However, more research with improved study design should be adopted to explore the mechanism associating AST and depressive symptoms.

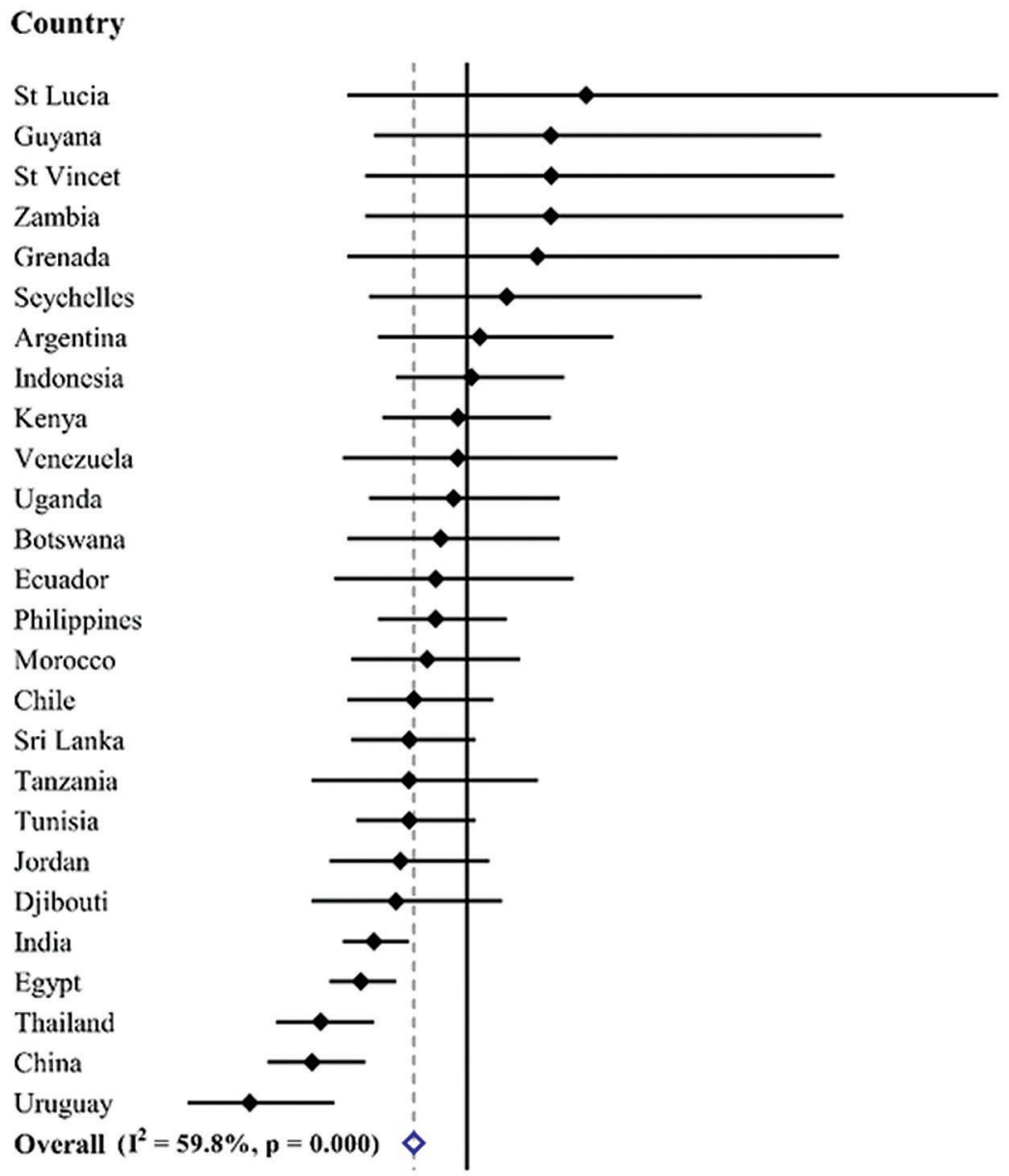

$\begin{array}{lll}\text { OR } & (95 \% \text { CI }) & \text { Weight } \\ 1.27 & (0.73,2.20) & 1.2 \\ 1.19 & (0.79,1.80) & 1.9 \\ 1.19 & (0.77,1.83) & 1.8 \\ 1.19 & (0.77,1.85) & 1.7 \\ 1.16 & (0.73,1.84) & 1.6 \\ 1.09 & (0.78,1.53) & 2.5 \\ 1.03 & (0.80,1.33) & 3.6 \\ 1.01 & (0.84,1.22) & 4.8 \\ 0.98 & (0.81,1.19) & 4.7 \\ 0.98 & (0.72,1.34) & 2.8 \\ 0.97 & (0.78,1.21) & 4.1 \\ 0.94 & (0.73,1.21) & 3.7 \\ 0.93 & (0.70,1.24) & 3.2 \\ 0.93 & (0.80,1.09) & 5.4 \\ 0.91 & (0.74,1.12) & 4.3 \\ 0.88 & (0.73,1.06) & 4.8 \\ 0.87 & (0.74,1.02) & 5.3 \\ 0.87 & (0.65,1.16) & 3.1 \\ 0.87 & (0.75,1.02) & 5.4 \\ 0.85 & (0.69,1.05) & 4.2 \\ 0.84 & (0.65,1.08) & 3.7 \\ 0.79 & (0.72,0.87) & 6.6 \\ 0.76 & (0.69,0.84) & 6.5 \\ 0.67 & (0.57,0.79) & 5.1 \\ 0.65 & (0.55,0.77) & 5.2 \\ 0.51 & (0.37,0.70) & 2.8 \\ \mathbf{0 . 8 8} & (0.82,0.94) & 100.0\end{array}$

Figure 1: Country-wise association between active school travel and depressive symptoms estimated by multivariable logistic regression. Note: $\mathrm{OR}=$ Odds ratio; $\mathrm{CI}=$ confidence interval; Models were adjusted for overall estimate was calculated by meta-analysis with random effects

Inconsistent with one previously published study [19], the present study found a significant negative relationship between AST and depressive symptoms irrespective of gender. A study by Gu et al. using similar measures of depressive symptoms to our study [19] found a significant association between AST and depression symptoms only existed among boys rather than girls. The discrepancy may be due to the cutoff of being active in school travel. In the current study, 5 days was adopted as a cutoff to categorize participants into either "active" or "passive" commuters whereas Gu et al. used 7 days [19]. This 
difference in measurement of being physically active in school travel may be the explanation for the different findings. Also, differences of sample characteristics of the two studies might also be another explanation. However, although the present study and that by Gu et al. [19] have contrary findings, more studies are required to explore whether the association between AST and depressive symptoms varies by gender. Another research finding in the present study was that the association between AST and depressive symptoms had a moderate inconsistency across different countries. In other words, the positive roles of AST on reducing depressive symptoms among adolescent varied greatly across the countries, which may be due to differences in socioeconomic status and cultural backgrounds. Therefore, it is worth noting that examining the role of AST in reducing depressive symptoms should be based on each country's situation. More specifically, it means that designing or implementing AST interventions to improve mental health, policymakers need to take into account specific circumstances and multiple factors in each country, such as built environmental (e.g., walk lanes, traffic problems) and social security.

We have to admit that owing to the nature of study design (cross-sectional) in the GSHS, the current study failed to examine the bidirectionality of the AST-depression association. Clearly, it is urgently needed to carry out longitudinal studies to determine if there is a casual association between AST and depressive symptoms in adolescents. Given the fact that depressive symptoms could result in lower levels of PA [33], which may reduce the possibility of attending AST as it is a component of PA. However, to the best of our knowledge, it remains largely unknown about which one is a precursor in terms of the AST-depression association among adolescents. This underpins the importance of clarifying direction of the association between AST and depressive symptoms.

As the current study found the negative association between AST and depressive symptoms in adolescents, promoting AST is an urgent required action plan.

\subsection{Limitation, Strengths and Future Research}

The research findings reported here should be interpreted in light of some limitations. First, the study was a cross-sectional investigation, which fails to provide true cause-and-effect association. Future longitudinal studies are required to further confirm the AST-depression association found in this age group or any other age group. Another limitation involves the measures for AST and depressive symptoms. For example, AST level was measured based on a single self-reported question and this approach failed to gain other important information (AST type [walking or cycling], distance between home and school, whether the way of travel is chosen or a given circumstance) that may also be closely associated with adolescent depression. Future studies are encouraged to explore the association between AST-related features (e.g., type, destination, and patter of AST) and mental health outcomes. Similarly, the measure for depressive symptoms was also a single self-reported question, with a sensitivity and specificity that remains unclear. Using this kind of measure can capture the information on depressive symptoms among adolescents quickly and easily but is subject to measurement error. Third, it is wellknown that depressive symptoms of adolescents are also influenced by some other factors including parental education or environmental variables, but they were not collected in the present study. This denotes the present study lacks some important covariates, which may cause errors in testing the research hypothesis. Fourth, our study did not include differences caused by survey years on study findings, which should be considered in future studies. Fifth, our study was based on samples from LMICs where adolescents are more likely to have higher crime rate and unwell-designed built environment for AST. Such real-world attributes may affect AST. Hence, future studies should consider these factors in examining the association between AST and mental health outcomes. Taken together, future studies should address the aforementioned limitations to provide more robust evidence.

However, the strengths of this study include large-size sample and data from a multinational perspective. Therefore, the research findings have wider generalizability, which could be applied in global depression 
prevention efforts among adolescents. Another strength of the study is that it is one of the very few studies exploring the relationship between AST and depressive symptoms among adolescents. This novelty is beneficial in developing new action plans or initiatives in preventing depression and reducing other mental or physical illnesses among young people. Given the preliminary evidence from the study, the role of AST impacting on mental health can be further expanded. Future public health interventions or initiatives should incorporate AST into health promotion plans for adolescent health development in respective contexts. Designing viable AST-promoting strategies may be also as effective in preventing depressive symptoms when possible and appropriate.

\section{Conclusions}

The present study offers multinational evidence on the association between active school travel and depressive symptoms among adolescents, indicating that adolescents with AST are less likely to have depressive symptoms irrespective of gender. However, this finding should be more fully explained based on each country's specific situations, including social and cultural factors. Based on the research findings, future study should attempt to confirm the roles of AST in promoting mental health among adolescents and explore further the underlying mechanisms. Study findings may provide researchers and policymakers to develop effective policies and action plans to decrease depressive symptoms among adolescents.

Acknowledgement: Thanks for all participants in this research project.

Authors' Contribution: SAL, STC, and LYZ conceived and designed the study; STC, LYZ, BS, QY, GM, and DZ analyzed data and interpreted the findings; STC and LYZ drafted this manuscript. All authors were involved in writing the paper, had final approval of the submitted and published version, and agree with authorship.

Funding Statement: Xiaohong Zhu is supported by the National Social Science Foundation (18BTY011). Brendon Stubbs is supported by a Clinical Lectureship (ICA-CL-2017-03-001) jointly funded by Health Education England (HEE) and the National Institute for Health Research (NIHR). Brendon Stubbs is part funded by the NIHR Biomedical Research Centre at South London and Maudsley NHS Foundation Trust. Brendon Stubbs also holds active grants with the Medical Research Council (GCRF and multimorbidity calls) and Guys and St Thomas Charity (GSTT). Brendon Stubbs has received consultancy fees from ASICS Europe BV. The views expressed are those of the author(s) and not necessarily those of the (partner organization), the NHS, the NIHR, the Department of Health and Social Care, the MRC or GSTT. ZD's contribution was supported by the Hungarian National Research, Development and Innovation Office (KKP126835; ELTE Thematic Excellence Programme 2020, KP2020-IKA-05).

Conflicts of Interest: The authors declare that they have no conflicts of interest to report regarding the present study.

\section{References}

1. Mehler-Wex, C., Kölch, M. (2008). Depression in children and adolescents. Deutsches Arzteblatt International, 105, 149-155. DOI 10.3238/arztebl.2008.0149.

2. Bernaras, E., Jaureguizar, J., Garaigordobil, M. (2019). Child and adolescent depression: A review of theories, evaluation instruments, prevention programs, and treatments. Frontiers in Psychology, 10, 49. DOI 10.3389/ fpsyg.2019.00543.

3. Balazs, J., Miklósi, M., Keresztény, Á., Apter, A., Bobes, J. et al. (2012). Prevalence of adolescent depression in Europe. European Psychiatry, 27(1), 259. DOI 10.1016/S0924-9338(12)74426-7.

4. Johnson, D., Dupuis, G., Piche, J., Clayborne, Z., Colman, I. (2018). Adult mental health outcomes of adolescent depression: A systematic review. Depression and Anxiety, 35(8), 700-716. DOI 10.1002/da.22777. 
5. Köhler, C. A., Evangelou, E., Stubbs, B., Solmi, M., Veronese, N. et al. (2018). Mapping risk factors for depression across the lifespan: An umbrella review of evidence from meta-analyses and Mendelian randomization studies. Journal of Psychiatric Research, 103(9), 189-207. DOI 10.1016/j.jpsychires.2018.05.020.

6. Oberste, M., Medele, M., Javelle, F., Wunram, H., Walter, D. et al. (2020). Physical activity for the treatment of adolescent depression: A systematic review and meta-analysis. Frontiers in Physiology, 11, 33. DOI 10.3389/ fphys.2020.00185.

7. Wegner, M., Amatriain-Fernandez, S., Kaulitzky, A., Murillo-Rodriguez, E., Machado, S. et al. (2020). Systematic review of meta-analyses: Exercise effects on depression in children and adolescents. Frontiers in Psychiatry, 11, 061024022229003. DOI 10.3389/fpsyt.2020.00081.

8. Artinian, N. T., Fletcher, G. F., Mozaffarian, D., Kris-Etherton, P., Van Horn, L. et al. (2010). Interventions to promote physical activity and dietary lifestyle changes for cardiovascular risk factor reduction in adults a scientific statement from the American Heart Association. Circulation, 122(4), 406-441. DOI 10.1161/ CIR.0b013e3181e8edf1.

9. Mammen, G., Faulkner, G. (2013). Physical activity and the prevention of depression: A systematic review of prospective studies. American Journal of Preventive Medicine, 45(5), 649-657. DOI 10.1016/j.amepre.2013.08.001.

10. Dale, L. P., Vanderloo, L., Moore, S., Faulkner, G. (2019). Physical activity and depression, anxiety, and selfesteem in children and youth: An umbrella systematic review. Mental Health and Physical Activity, 16(4), 66-79. DOI 10.1016/j.mhpa.2018.12.001.

11. Kleppang, A. L., Hartz, I., Thurston, M., Hagquist, C. (2018). The association between physical activity and symptoms of depression in different contexts-a cross-sectional study of Norwegian adolescents. BMC Public Health, 18(1), 403. DOI 10.1186/s12889-018-6257-0.

12. Belair, M. A., Kohen, D. E., Kingsbury, M., Colman, I. (2018). Relationship between leisure time physical activity, sedentary behaviour and symptoms of depression and anxiety: Evidence from a population-based sample of Canadian adolescents. BMJ Open, 8(10), e021119. DOI 10.1136/bmjopen-2017-021119.

13. Kandola, A., Lewis, G., Osborn, D. P. J., Stubbs, B., Hayes, J. F. (2020). Depressive symptoms and objectively measured physical activity and sedentary behaviour throughout adolescence: A prospective cohort study. Lancet Psychiatry, 7(3), 262-271. DOI 10.1016/S2215-0366(20)30034-1.

14. Brusseau, T., Fairclough, S., Lubans, D. (2020). The routledge handbook of youth physical activity. UK: Routledge.

15. Guell, C., Ogilvie, D. (2014). Picturing commuting: Photovoice and seeking well-being in everyday travel. Qualitative Research, 15(2), 201-218. DOI 10.1177/1468794112468472.

16. Hiscock, R., Macintyre, S., Kearns, A., Ellaway, A. (2002). Means of transport and ontological security: Do cars provide psycho-social benefits to their users? Transportation Research Part D: Transport and Environment, 7(2), 119-135. DOI 10.1016/S1361-9209(01)00015-3.

17. Waygood, E. O. D., Friman, M., Olsson, L. E., Taniguchi, A. (2017). Transport and child well-being: An integrative review. Travel Behaviour and Society, 9(55), 32-49. DOI 10.1016/j.tbs.2017.04.005.

18. Schoeppe, S., Duncan, M. J., Badland, H., Oliver, M., Curtis, C. (2013). Associations of children's independent mobility and active travel with physical activity, sedentary behaviour and weight status: A systematic review. Journal of Science and Medicine in Sport, 16(4), 312-319. DOI 10.1016/j.jsams.2012.11.001.

19. Gu, J., Chen, S. T. (2020). Association between active travel to school and depressive symptoms among early adolescents. Children, 7(5), 41. DOI 10.3390/children7050041.

20. Babey, S. H., Hastert, T. A., Huang, W., Brown, E. R. (2009). Sociodemographic, family, and environmental factors associated with active commuting to school among US adolescents. Journal of Public Health Policy, 30(1), 203-220. DOI 10.1057/jphp.2008.61.

21. Chillón, P., Ortega, F. B., Ruiz, J. R., Pérez, I. J., Martín-Matillas, M. et al. (2009). Socio-economic factors and active commuting to school in urban Spanish adolescents: The AVENA study. European Journal of Public Health, 19(5), 470-476. DOI 10.1093/eurpub/ckp048.

22. Pinto, A. D. A., Claumann, G. S., Angelo, H. C. C. D., Menezes, E. C., Dias, D. T. et al. (2017). Active commuting to school and associated factors among adolescents: A Systematic Review. Journal of Physical Education, 28(1), e2859. DOI 10.4025/jphyseduc.v28i1.2859. 
23. Timperio, A., Ball, K., Salmon, J., Roberts, R., Giles-Corti, B. et al. (2006). Personal, family, social, and environmental correlates of active commuting to school. American Journal of Preventive Medicine, 30(1), 45-51. DOI 10.1016/j.amepre.2005.08.047.

24. Sun, Y., Liu, Y., Tao, F. B. (2015). Associations between active commuting to school, body fat, and mental wellbeing: Population-based, cross-sectional study in China. Journal of Adolescent Health, 57(6), 679-685. DOI 10.1016/j.jadohealth.2015.09.002.

25. Ramanathan, S., O'Brien, C., Faulkner, G., Stone, M. (2014). Happiness in motion: Emotions, well-being, and active school travel. Journal of School Health, 84(8), 516-523. DOI 10.1111/josh.12172.

26. Vancampfort, D., Stubbs, B., Firth, J., van Damme, T., Koyanagi, A. (2018). Sedentary behavior and depressive symptoms among 67,077 adolescents aged 12-15 years from 30 low- and middle-income countries. International Journal of Behavioral Nutrition and Physical Activity, 15(1), 1575. DOI 10.1186/s12966-018-0708-y.

27. Chen, S. T., Guo, T., Yu, Q., Stubbs, B., Clark, C. et al. (2021). Active school travel is associated with fewer suicide attempts among adolescents from low-and middle-income countries. International Journal of Clinical and Health Psychology, 21(1), 100202. DOI 10.1016/j.ijchp.2020.11.001.

28. Prochaska, J. J., Sallis, J. F., Long, B. (2001). A physical activity screening measure for use with adolescents in primary care. Archives of Pediatrics \& Adolescent Medicine, 155(5), 554-559. DOI 10.1001/archpedi.155.5.554.

29. Higgins, J. P., Thompson, S. G. (2002). Quantifying heterogeneity in a meta-analysis. Statistical Medicine, 21(11), 1539-1558. DOI 10.1002/(ISSN)1097-0258.

30. Biddle, S. J. H., Ciaccioni, S., Thomas, G., Vergeer, I. (2019). Physical activity and mental health in children and adolescents: An updated review of reviews and an analysis of causality. Psychology of Sport and Exercise, 42(4), 146-155. DOI 10.1016/j.psychsport.2018.08.011.

31. Fusco, C., Moola, F., Faulkner, G., Buliung, R., Richichi, V. (2012). Toward an understanding of children's perceptions of their transport geographies: (non) active school travel and visual representations of the built environment. Journal of Transport Geography, 20(1), 62-70. DOI 10.1016/j.jtrangeo.2011.07.001.

32. Lambiase, M. J., Barry, H. M., Roemmich, J. N. (2010). Effect of a simulated active commute to school on cardiovascular stress reactivity. Medicine \& Science in Sports \& Exercise, 42(8), 1609-1616. DOI 10.1249/ MSS.0b013e3181d0c77b.

33. de Geus, E. J. C. (2021). A genetic perspective on the association between exercise and mental health in the era of genome-wide association studies. Mental Health and Physical Activity, 20(7), 100378. DOI 10.1016/j. mhpa.2020.100378. 